\title{
Is heart rate response a reliable marker of adenosine-induced coronary hyperemia?
}

\author{
Bhavik N. Modi ${ }^{1} \cdot$ Haseeb Rahman ${ }^{1} \cdot$ Sara Abou Sherif $^{1} \cdot$ Howard Ellis $^{1} \cdot$ Kseniia Eruslanova $^{1} \cdot$ Amedeo Chiribiri ${ }^{1}$. \\ Divaka Perera ${ }^{1}$
}

Received: 12 December 2017 / Accepted: 31 January 2018 / Published online: 14 February 2018

(c) The Author(s) 2018. This article is an open access publication

\begin{abstract}
Introduction Growing evidence supports ischemia-guided management of chest pain, with invasive and non-invasive tests reliant upon achieving adenosine-induced coronary hyperemia (defined as increased blood flow to an organ's perfusion bed). In the non-invasive setting, surrogate markers of hyperemia, such as increases in heart rate, are often used, despite not being formally validated. We tested whether heart rate and other non-invasive indices are reliable markers of coronary hyperemia. Methods The first part involved Doppler flow-based validation of the best pressure-wire markers of hyperemia in 53 patients. Subsequently, using these validated pressure-derived parameters, 265 pressure-wire traces were analysed to determine whether heart rate and other non-invasive parameters correlated with hyperemia. Results In the flow derivation cohort, the best determinant of hyperemia came from having 2 out of 3 of: (1) Ventriculisation of the distal pressure waveform, (2) disappearance of distal dicrotic pressure notch, (3) separation of mean aortic and distal pressures. Within the 244 patients demonstrating hyperemia, non-invasive markers of hyperemia, such as change in heart rate $(p=0.77)$, blood pressure $(p=0.60)$ and rate-pressure product $(p=0.86)$, were poor correlates of coronary hyperemia, with only $37.3 \%$ demonstrating $\mathrm{a} \geq 10 \%$ increase in heart rate that is commonly used to adjudge adenosine-induced hyperemia in the non-invasive setting. Conclusions We demonstrate, by correlation with Doppler-flow data, a validated method of identifying coronary hyperemia within the catheter laboratory using the pressure-wire. We subsequently show that non-invasive parameters, such as heart rate change, are poor predictors of coronary hyperemia during stress imaging protocols that rely upon achieving adenosineinduced hyperemia.
\end{abstract}

Keywords Adenosine $\cdot$ Hyperemia $\cdot$ Fractional flow reserve $\cdot$ Stress perfusion cardiac MRI $\cdot$ Coronary artery disease

\section{Introduction}

A growing body of evidence supports ischemia-guided revascularisation [1]. Surrogates of ischaemia can be assessed non-invasively or during diagnostic angiography, often relying on pharmacological induction of coronary hyperemia [2]. The most widely used invasive measure is fractional flow reserve (FFR) [3-5], based on the measurement of distal coronary and aortic pressure during adenosine-induced hyperemia. Intravenous (IV) adenosine is also

Divaka Perera

Divaka.Perera@kcl.ac.uk

1 NIHR Biomedical Research Centre and British Heart Foundation Centre of Excellence, School of Cardiovascular Medicine and Sciences, St Thomas' Campus, King's College London, London, UK used in stress perfusion cardiac magnetic resonance imaging (CMR). This test is increasingly used to detect and quantify ischaemia in patients with suspected coronary disease [6] by demonstrating regional heterogeneity of coronary blood flow during hyperemia. Intravenous adenosine at a dose of $140 \mathrm{mcg} / \mathrm{kg} / \mathrm{min}$, has been shown to reliably induce nearmaximal hyperemia in most patients, with minimal sideeffects [7]. The net effect of IV adenosine in humans is typically a mild reduction in arterial blood pressure associated with increases in heart rate (HR), with multiple mechanisms proposed [7, 8]. Due to its non-selectivity, adenosine also activates other receptors $\left(\mathrm{A}_{1}, \mathrm{~A}_{2 \mathrm{~B}}\right.$ and $\left.\mathrm{A}_{3}\right)$, which can also result in cardiac conduction abnormalities, hypotension, flushing and bronchospasm [9].

True hyperemia is best assessed by showing increases in coronary blood flow measured invasively using Doppler or thermodilution techniques, that are difficult to implement 
outside the research setting. Predicting when a patient is experiencing maximal hyperemia within the catheter laboratory is therefore sometimes assessed by awaiting the onset of flushing, breathlessness and chest tightness symptoms. Additionally, non-invasive surrogates such as blood pressure drop, HR rise and changes in aortic and distal coronary pressure waveforms are relied upon to determine the onset of hyperemia, although no reproducible and objective criteria have been identified.

Within the non-invasive CMR setting, where it is not possible to measure such invasive indices, subjective symptoms along with objective hemodynamic measures of increasing HR and falling systolic blood pressure (SBP) are used as surrogate markers of hyperemia. $10 \%$ or 10 beats per minute increase in HR, is commonly considered a marker of adequate hyperemia within the imaging setting, its absence thought to imply inadequate hyperemic stimulus. In these cases, higher adenosine doses are administered or the study is classified as equivocal [10]. A sub-analysis of the CEMARC study suggests that inadequate hyperemic response is considered a recognized cause of a false-negative CMR perfusion scan [11].

In this study, we tested the hypothesis that HR changes, and other surrogate non-invasive indices are reliable markers of coronary hyperemia.

\section{Methods}

\section{Study population}

Our study population consisted of patients who presented to a single centre for coronary angiography \pm proceeding to percutaneous intervention as appropriate. 306 Consecutive patients undergoing FFR measurements between October 2013 and February 2017 were screened, where hyperemia was induced by IV adenosine infusion. Between this period, 53 patients also had simultaneous pressure and Doppler measurements using a CombowireXT guidewire (Philips Volcano) as part of a number of studies utilizing detailed intracoronary physiological measurements in patients with ischemic heart disease. All patients received an IV adenosine infusion dose of $140 \mathrm{mcg} / \mathrm{kg} / \mathrm{min}$ through an antecubital vein using a standardized infusion pump at a fixed distance from the patient, to minimize variability. For the purposes analysis, FFR was defined as the lowest $\mathrm{Pd} / \mathrm{Pa}$ ratio following the onset of adenosine, averaged over five cardiac cycles, also known as the 'smart minimum FFR' [12]. All participants gave written informed consent in accordance with the protocol approved by the local research ethics committee. The study protocol conformed to the ethical guidelines of the 1975 Declaration of Helsinki.

\section{Standardisation of invasive pressure assessment of hyperemia}

Hyperemia was assessed in the 53 patients using Doppler flow velocity measurements, by examining coronary flow reserve (CFR), defined as the ratio of average peak flow velocity (APV) compared to baseline. Doppler measurements of coronary blood flow velocity have been shown to have inter- and intra-observer variability of approximately $10 \%$ [13]. A pre-defined CFR threshold of 1.2 was therefore used to define hyperemia (defined as an increase in blood flow to an organ's perfusion bed) to ensure that the increase in flow at hyperemia is above the margin of measurement error commonly seen with CombowireXT Doppler flow measurements.

In this Doppler cohort, we assessed the diagnostic performance of three commonly-used invasive pressure-waveform parameters of hyperemia, and combinations thereof by calculating their sensitivity, specificity and positive and negative predictive values. These are (1) ventricularisation of distal pressure waveform (a presystolic deflection resembling an 'a wave', a slower upstroke of the waveform and a steeper down-stroke than that of aortic pressure [14]), (2) separation of mean aortic and distal coronary pressure [ $>10 \%$ difference in ( $\mathrm{Pa}-\mathrm{Pd})$, over five consecutive heart beats, compared to the resting gradient] and (3) disappearance of dicrotic notch from the distal arterial pressure trace (see Fig. 1). In the absence of an established CFR cut-off for defining hyperemia, the diagnostic performance of the pressure-based parameters at CFR of 1.2 were also compared to their performance at a higher CFR of 1.5.

\section{Assessing adenosine-mediated changes in coronary microvascular resistance and peripheral vascular resistance}

The flow cohort enabled characterization of coronary microvasculature resistance (MR) and peripheral vascular resistance (via the augmentation index, AIx) [15], calculated over five consecutive beats at rest and hyperemia. MR was defined as the distal pressure divided by APV. AIx; a measure of central aortic pressure-waveform enhancement by a reflected pulse wave, is calculated as the difference between this late systolic pressure $P_{2}$ and early systolic pressure $P_{l}$, as a percentage of pulse pressure, whereby $P_{1}$ was identified as the first peak on an aortic pressure wave (resulting from the ejection of blood from the heart) and $P_{2}$ identified as the second peak (resulting from reflection of blood due to constriction downstream in the peripheral vascular tree). 


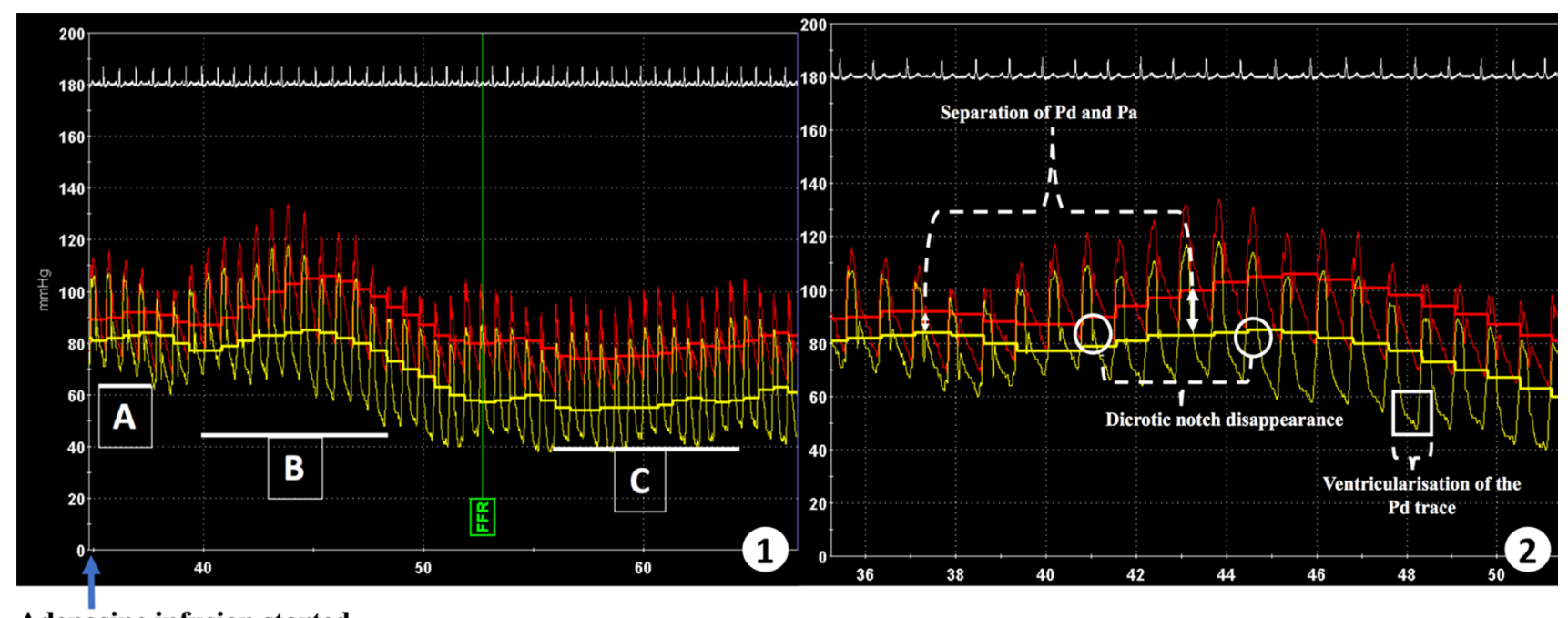

Adenosine infusion started

Fig. 1 Pressure-Derived Invasive Parameters. 1 The three-invasive pressure-based parameters that were investigated $(A, B$ and $C)$ and subsequently used to define hyperemia during IV adenosine infusion.

\section{Assessing diagnostic performance of heart rate and other non-invasive surrogate markers of hyperemia}

Based on the diagnostic performance of the invasive pressure parameters (and combinations thereof) in the Doppler cohort, the study population $(\mathrm{n}=265)$ were dichotomously classified as hyperemic or non-hyperemic. The predictive accuracy of commonly used non-invasive haemodynamic markers [HR, SBP, and rate pressure product (RPP, $\mathrm{HR} \times \mathrm{SBP})]$ were analysed as a percentage change in each parameter in response to IV adenosine-induced hyperemia.

\section{Statistical analysis}

Continuous variables were assessed for normality and if found to be normally distributed, were expressed as mean \pm standard deviation. Categorical variables were expressed as counts and percentages. Differences in continuous variables were assessed by an independent Student's $t$ test, whilst differences in categorical variables were evaluated by Fisher's exact Chi-Squared test. Differences in continuous matched variables, such as HR at rest and at hyperemia were assessed using a Paired $t$ test. The diagnostic accuracy of invasive pressure-waveform parameters at detecting a CFR $>1.2$ was classified in terms of specificity, sensitivity, positive predictive value (PPV) and negative predictive value (NPV). Changes in microvascular resistance and peripheral vascular resistance were correlated using Pearson's Rank Correlation, after testing for normality, and results reported a $R^{2}$ values. Statistical analyses were
Red trace $=\mathrm{Pa}$ (aortic wave), yellow trace $=\mathrm{Pd}$ (distal coronary wave). 2 Magnification of the three-invasive pressure-bounded parameters

performed using SPSS software (version 22.0; SPSS Inc., Chicago, IL, USA).

\section{Results}

\section{Performance of invasive pressure-indices of hyperemia}

In the 53 patients with simultaneous pressure and Doppler measurements, mean FFR was $0.84 \pm 0.1$. The individual pressure-waveform indices all had good sensitivity at detecting hyperemia, defined using a CFR threshold of 1.2 , but relatively low specificity. Disappearance of the dicrotic notch and ventricularisation of the distal pressure waveform were the two parameters achieving the best NPV and PPV (Fig. 2; Table 1). The reduced diagnostic performance of Pd and Pa trace separation (Fig. 2), can be explained by the fact that this parameter is difficult to detect in normal coronary arteries (FFR $>0.9$ ). In contrast dicrotic notch disappearance and ventricularisation were detected easily, regardless of disease burden. Combining these indices improved specificity and hence the presence of at least $2 / 3$ pressure indices was chosen as the optimum criterion for detecting hyperemia, forming the basis of detecting hyperemia in the pressure cohort.

When a higher CFR threshold of 1.5 was used, the diagnostic performance of the pressure-bounded parameters was almost identical to a CFR of 1.2. However, by using a CFR threshold of 1.5, hyperemic and non-hyperemic 


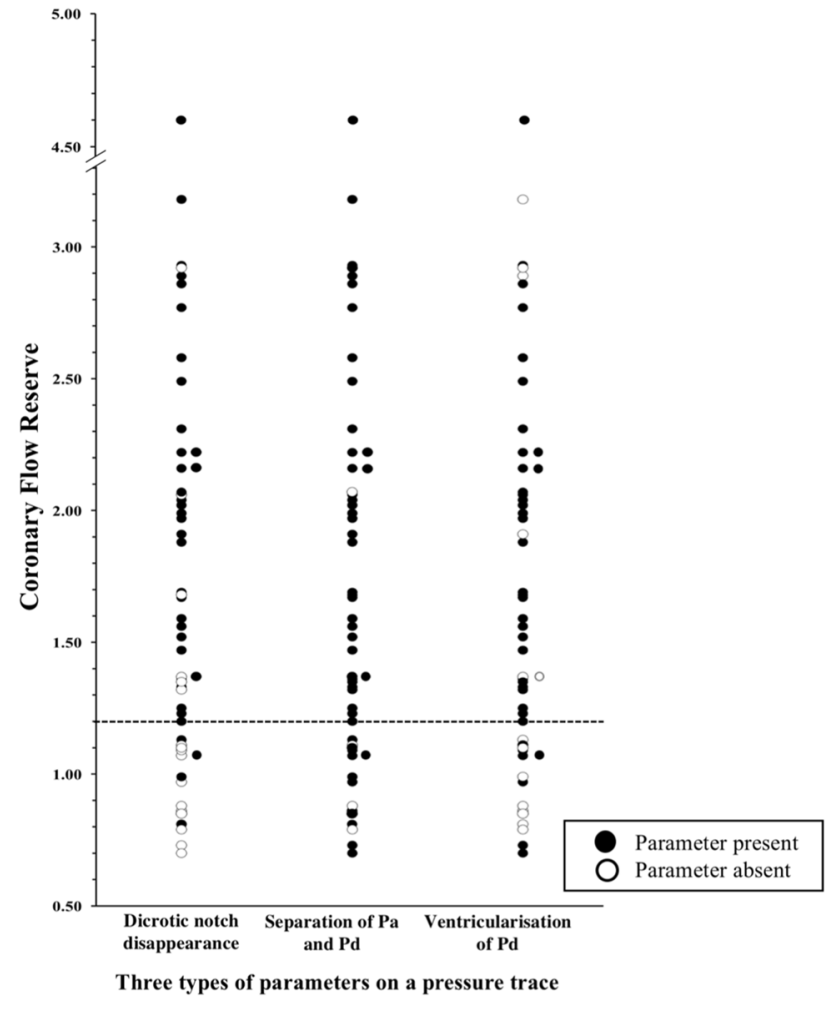

Fig. 2 Relationship between coronary flow reserve (CFR) and the three invasive parameters of hyperemia: graph illustrating the presence and absence of pressure-based parameters of hyperemia in 53 patients where simultaneous CFR value were measured. CFR cut-off of 1.2 used as a marker of definitive hyperemia

rates were found to be clinically unrealistic at 53 and $47 \%$ respectively.

\section{Changes in coronary microvascular resistance and peripheral vascular resistance}

Using flow data from 53 patients, we found both the MR and AIx significantly dropped from rest to hyperemia $(-37 \pm 29.76 \%, p<0.001$ and $-7.13 \pm 55.32 \%, p=0.004)$.
There was no correlation between HR change and AIx, $\mathrm{R}^{2}=0.031, \mathrm{p}=0.2$ (2-sided), or between changes in $\mathrm{MR}$ and AIx from rest, $\mathrm{R}^{2}=0.021, \mathrm{p}=0.3$.

\section{Dichotomization of patients to hyperemic and non-hyperemic}

306 Consecutive patients undergoing invasive FFR measurements between October 2013 and February 2017 were screened, where hyperemia was induced by IV infusion of adenosine. 265 patients were analysed; 41 patients were excluded for reasons specified in the study flow chart (Fig. 3). Based on our validated pressure-based criteria, 244 of the 265 patients were determined to have developed hyperemia.

\section{Patient characteristics}

The enrolled population of 265 patients was $65 \pm 11$ years old with $74 \%$ male. We assessed one vessel per patient and found the mean FFR, in cases where hyperemia was adjudged to have been reached, was $0.81 \pm 0.09$. Although the proportion of patients with previous PCI was higher patients determined to have reached hyperemia compared to those that did not, there were no other significant differences in patient characteristics between the hyperemic and non-hyperemic groups (Table 2).

\section{Assessment of heart rate and other non-invasive surrogate markers of hyperemia}

The percentage change in HR from rest did not differ significantly between hyperemic and non-hyperemic groups; $7.9 \pm 14.0$ and $7.0 \pm 16.3$ respectively $(p=0.78)$. In addition, there was no significant difference in the proportion of patients exhibiting $\mathrm{a} \geq 10 \%$ increase in HR between these groups; 37 vs. $34 \%$, p =0.10 (Fig. 4). Similarly, when these non-invasive parameters were assessed in the 53 flow-data cohort, there was no significant difference in HR, RPP and SBP from rest between hyperemic and non-hyperemic
Table 1 Diagnostic performance of each invasive parameter, and combinations of 2 , at a CFR threshold of 1.2

\begin{tabular}{lllll}
\hline & Sens & Spec & NPV & PPV \\
\hline Dicrotic notch disappearance & 84.2 & 73.3 & 88.9 & 64.7 \\
Separation of Pa and Pd & 97.4 & 20 & 75.5 & 75 \\
Ventricularisation of Pd trace & 84.2 & 53.3 & 82.1 & 57.1 \\
Dicrotic notch disappearance + ventricularisation & 73.7 & 93.3 & 96.6 & 58.3 \\
Dicrotic notch disappearance + separation of Pa and Pd & 81.6 & 73.3 & 88.6 & 61.1 \\
Separation of Pa and Pd + ventricularisation & 81.6 & 60 & 83.8 & 56.3 \\
\hline
\end{tabular}

Within the validation cohort of patients with pressure and flow data, a CFR cut-off of 1.2 was used to calculate sensitivity (Sens), specificity (Spec), negative predictive (NPV) and positive predictive value (PPV) of the three pressure based parameters. Diagnostic performance was assessed both on their own and/or in different paired combinations 


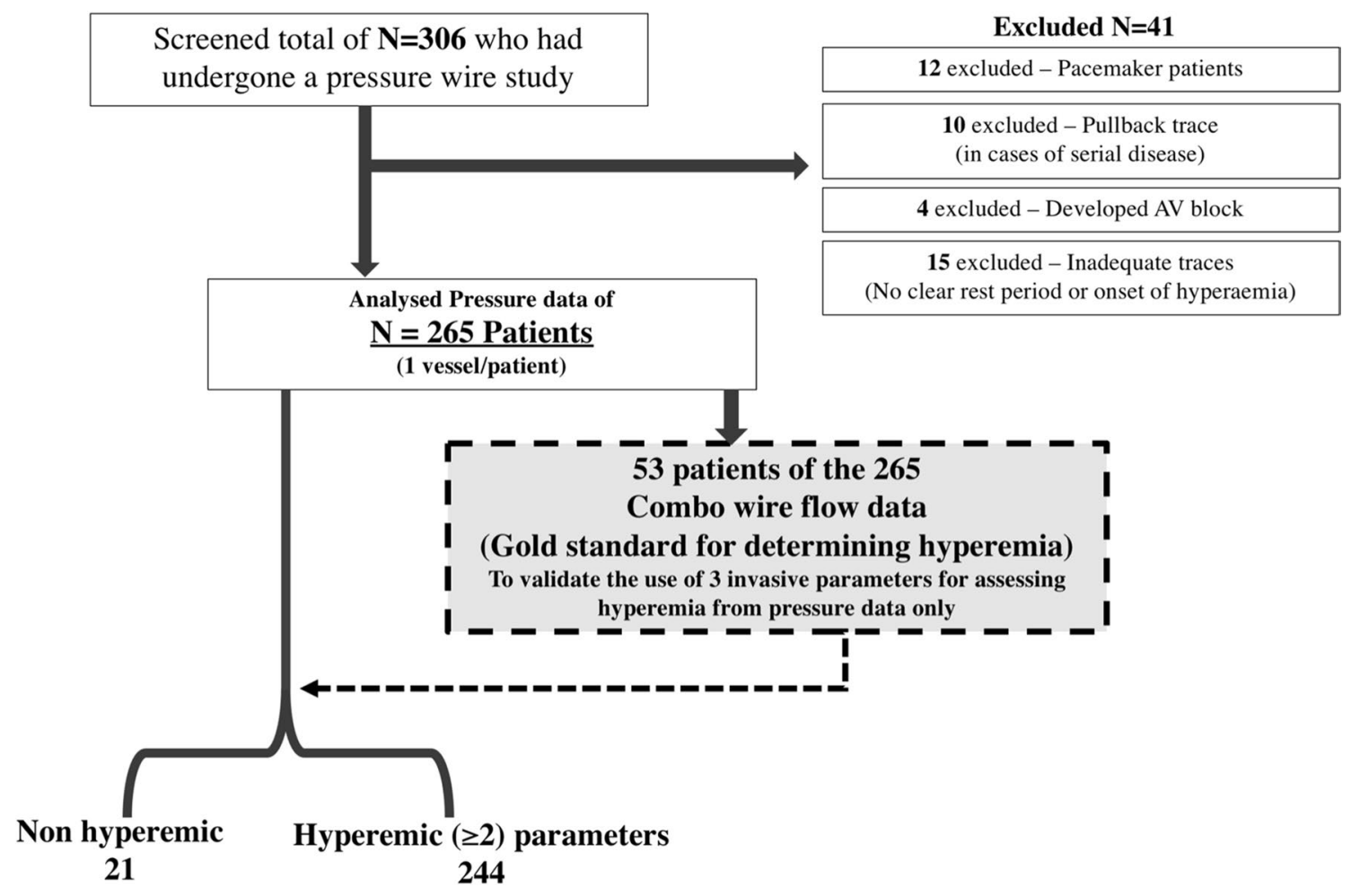

Fig. 3 Flow chart: 265 patients with pressure data were analysed using the flow-validated pressure indices to determine hyperemic or not. The predictive accuracy of commonly used non-invasive haemo-

$H R \times S B P$ rate pressure product) were analysed as \% change in each dynamic markers ( $H R$ heart rate, $S B P$ systolic blood pressure and parameter in response to IV adenosine from rest to the onset of the lowest $\mathrm{Pd} / \mathrm{Pa}$ ratio

Table 2 Demographics of patient population

\begin{tabular}{lllllll}
\hline & \multicolumn{2}{l}{ Hyperemic } & & \multicolumn{2}{l}{ Non hyperemic } & p value \\
\cline { 2 - 3 } Variables & $\mathrm{N}=244$ & $\%$ & & $\mathrm{~N}=21$ & $\%$ & \\
\hline Age & $65 \pm 10.8$ & & & $67 \pm 9.7$ & & 0.63 \\
Sex (M/F) & $181 / 63$ & $74.2 / 25.8$ & & $16 / 5$ & $76.2 / 23.8$ & 0.54 \\
Hypertension & 152 & 62.3 & & 10 & 47.6 & 0.14 \\
Hypercholesterolemia & 181 & 74.2 & & 16 & 76.2 & 0.54 \\
Diabetes mellitus & 59 & 24.2 & & 8 & 38.1 & 0.13 \\
Smoker & 49 & 20.1 & & 4 & 19 & 0.59 \\
Patients with a history of MI & 61 & 25 & & 3 & 14.3 & 0.21 \\
Patients with a history of PCI & 90 & 36.9 & & 3 & 14.3 & 0.03 \\
Patients with a history of CABG & 10 & 4.1 & & 0 & 0 & \\
Indication for PCI: stable elective & 221 & 90.6 & & 20 & 95.2 & 0.41 \\
Indication for PCI: ACS & 23 & 9.4 & & 1 & 4.8 & 0.41 \\
\hline
\end{tabular}

Comparison of demographics in hyperemic and non-hyperemic groups

$M$ male, $F$ female, $M I$ myocardial infarction, $P C I$ percutaneous coronary intervention, $C A B G$ coronary artery bypass graft, $A C S$ acute coronary syndrome

*p value calculated by independent samples $t$ test for age variable and Chi-squared significance for remaining variables patients, as defined by flow. Of the 38 hyperemic individuals (as defined by CFR $\geq 1.2$ ), only $47 \%$ showed $\mathrm{a} \geq 10 \%$ increase in HR. Overall, assessment of the diagnostic performance of HR in the validation cohort revealed a sensitivity of $37.3 \%$, specificity of $81 \%$, PPV of $96 \%$ and NPV of $10 \%$. Similarly, when assessing HR in the flow-cohort, 


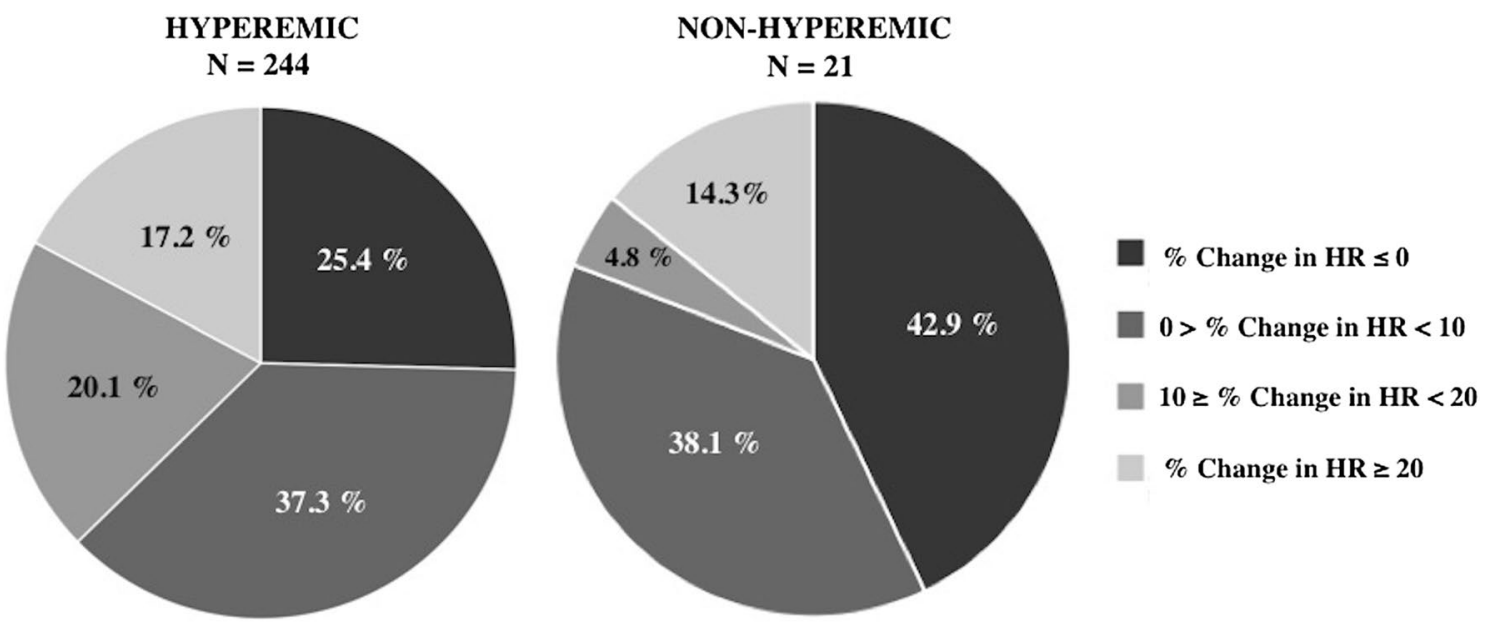

Fig. 4 Heart rate variability. Pie chart representation of variability in percentage change in HR in patients deemed to be hyperemic versus those that were not

Fig. 5 Variability in heart rate and $\mathrm{Pd} / \mathrm{Pa}$ over time. An illustration of how the mean HR and Pd-Pa changed over the course of adenosine infusion in 244 hyperemic patients

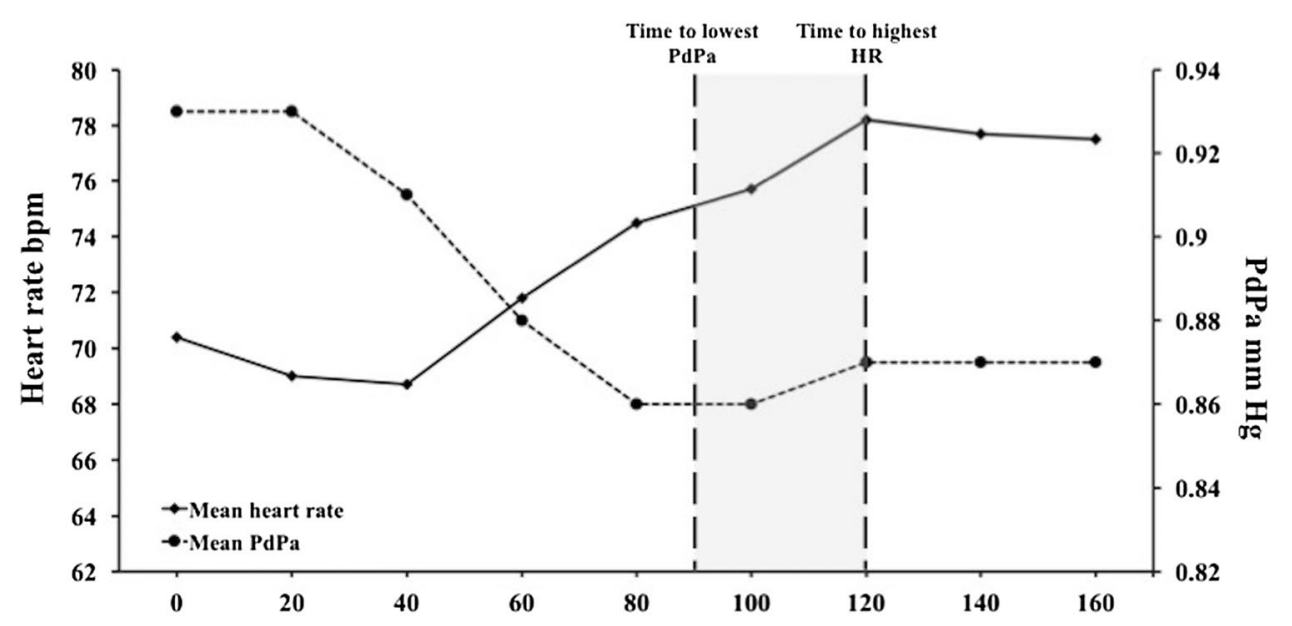

Time from start of IV adenosine infusion (sec)

where hyperemia was determined using direct Doppler measurements, HR showed a sensitivity of $47.4 \%$, specificity of $86.7 \%$, PPV of $90 \%$ and NPV of $39.4 \%$. In patients where hyperemia was achieved, the lowest $\mathrm{Pd} / \mathrm{Pa}$ value occurred at $87.8 \pm 32.6 \mathrm{~s}$ : significantly earlier than the mean time to peak HR (Fig. 5). Other commonly used non-invasive surrogate markers, such as SBP and RPP, also did not vary significantly between hyperemic and non-hyperemic patients (Fig. 6).

\section{Discussion}

In this study, we have demonstrated that the tachycardia associated with IV adenosine infusion is an unreliable surrogate marker of maximal hyperemia, with only $37.3 \%$ hyperemic patients exhibiting a 10\% increase in HR. This has important implications during non-invasive testing, such as perfusion CMR, when a $10 \%$ (or $10 \mathrm{bpm}$ ) increase in mean HR is often used as a marker of hyperemia, although there is no consensus method recommended in the guidelines $[10,16]$.

The first part of this study demonstrated the best performance for assessing hyperemia came from having $2 / 3$ of: (1) Ventriculisation of distal pressure waveform, (2) disappearance of distal dicrotic pressure-waveform notch, (3) separation of aortic and distal mean pressures (see Fig. 2; Table 1). Using $\geq 2 / 3$ of these parameters, we found $8 \%$ of patients did not exhibit a hyperemic response. Other studies measuring coronary flow during IV adenosine administration found similar rates of submaximal blood flow [7, $17,18]$, however studies that adjudged hyperemia by only 

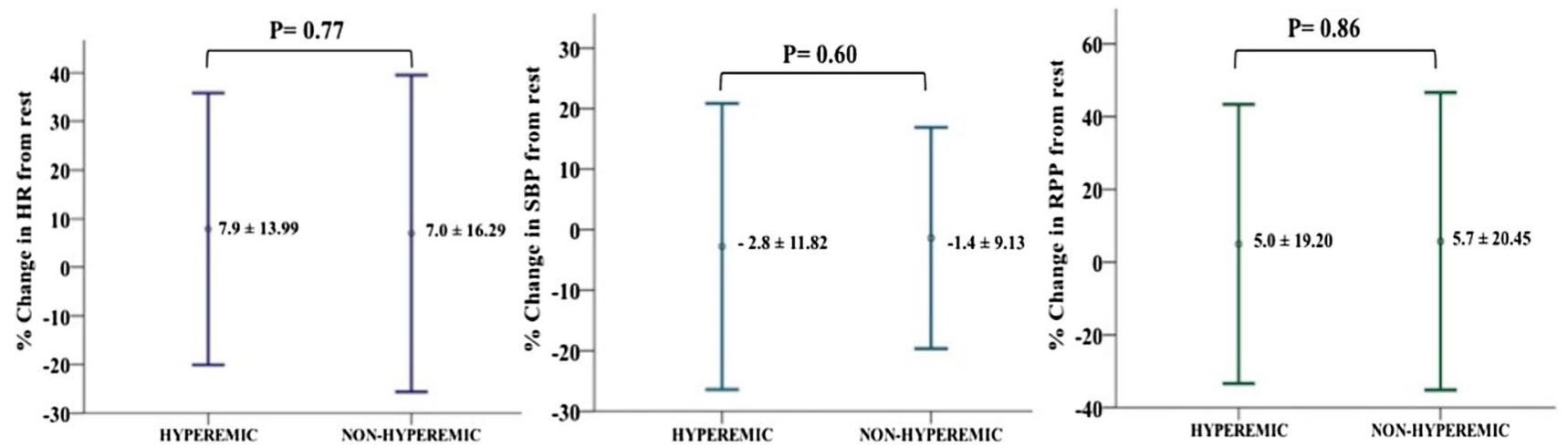

Fig. 6 Variability of non-invasive surrogate markers of hyperemia: a comparison of haemodynamic markers (RPP, HR and SBP) between patients achieving hyperemia and those that did not. Values are quoted as means \pm standard deviation

demonstrating separation between $\mathrm{Pd}$ and $\mathrm{Pa}$ traces found higher rates of 'non-hyperemic response to IV adenosine' [18]. We demonstrated that this parameter by itself poorly discriminates true hyperemia (Fig. 2), unsurprising in cases of minimal stenoses, where appreciable drops in $\mathrm{Pd} / \mathrm{Pa}$, even during hyperemia, may not occur [19]. A CFR of 1.5 was also assessed and whilst similar diagnostic performance was found, the resulting rate of hyperemia was only $52.8 \%$ which does not reflect a realistic response to adenosine in the clinical setting, nor does it coincide with the rates of hyperemia reported in previously. Whilst it may be surprising that in the derivation cohort of 53 patients, around half had CFR $<1.5$, this group of patients were pre-selected to consist of an intermediate coronary artery disease severity, whereby associated microvascular dysfunction may have contributed to overall reduced flow augmentation following adenosine administration. A CFR cut-off of 1.2 was therefore used to not only compensate for the classical $10-15 \%$ error of Doppler measurements, but also to yield clinically realistic PPV, NPV and hyperemia rates, compatible with previous reports [7].

Nearly two-thirds of patients with demonstrable hyperemia did not have an accompanying $\geq 10 \%$ HR increase commonly used to infer hyperemia non-invasively. Consequently, the NPV of HR was found to be poor. The poor performance of HR in the flow-defined group further supports its unreliability as a surrogate marker of hyperemia. Had this arbiter been applied in the perfusion MRI setting, these patients would have been misclassified as "non-responders", potentially leading to unnecessarily higher doses of adenosine (with more unpleasant side-effects) without added diagnostic value or misclassifying negative perfusion scans as equivocal $[7,10,20]$. We have also shown that the time-course of HR change may not reflect the onset of maximal hyperemia (Fig. 5), with prolonged adenosine infusions subjecting patients to unpleasant symptoms unnecessarily. Interestingly, we found $12.3 \%$ of patients showed a phasic response to
IV adenosine; whilst lower than the $39 \%$ previously quoted [12], if first pass perfusion image acquisition occurs during the inter-hyperemic window, the diagnostic value of the scan may be compromised, leading to further diagnostic inaccuracy when using IV adenosine in the non-invasive setting.

\section{Potential mechanisms underlying variation heart rate response to adenosine}

The ability of adenosine to induce tachycardia is ascribed to peripheral vasodilatation, with the assumption that HR change is a direct reflection of peripheral vasodilatation. In addition, both direct and reflex baroreceptor-mediated sympathetic activation are thought to play a role [21, 22]. Our study has shown that there was no correlation between HR, MR and AIx, and therefore highlights the likelihood of other mechanisms, beyond peripheral vasodilatation, by which HR increases in response to adenosine; such as action on the sympathetic nervous system [23]. The lack of correlation between HR and peripheral vasodilatation could also be explained by variable peripheral vasodilatory responses and known variations within adenosine receptor signalling pathways across individuals [23, 24].

Even in the presence of a significant HR increase, mean peak HR and mean lowest $\mathrm{Pd} / \mathrm{Pa}$ occurred at different times (Fig. 5). If peripheral vasodilation is the major determinant of HR increases, this response may be captured at a different time frame to that of maximal coronary vasodilatation and it could be inferred that complete saturation of the coronary and peripheral vascular beds may be occurring at separate times during adenosine infusion. This is supported by the fact that adenosine receptors have been found to vary in affinity for drugs across different vascular beds [25, 26].

If HR is an unreliable surrogate of hyperemia then what other options exist in non-invasive settings, such as CMR? Our data suggests that SBP or RPP changes are similarly unreliable indices of hyperemia (Fig. 6). Another option is to focus 
on non-haemodynamic markers of hyperemia such as splenic blood flow attenuation, which can be assessed during a single breath-hold without the need for gadolinium and may be a more reliable marker of coronary hyperemia compared to classical haemodynamic markers such as change in HR or SBP [27]. Another potential method of limiting the HR variability is to consider further investigating vasodilator agents with more selective A2A-receptor action within the non-invasive imaging setting, such as Regadenoson [28].

Our study has demonstrated that assessment of hyperemia in the cardiac catheterisation laboratory should rely on a combination of several invasive pressure-waveform based indices. In the non-invasive CMR setting, we demonstrate that HR and other haemodynamic surrogates are unreliable markers of hyperemia, owing perhaps to variability in dose responses in different vascular beds. Although our study did not specifically evaluate variability in symptoms during adenosine-induced hyperemia, anecdotal variability, e.g. chest tightness, breathlessness and flushing, are also likely to be a result of variability in adenosine receptor responses in different vascular beds. Whilst we appreciate that symptomatic changes are important to assess, due to the retrospective nature of the study, we could not assess these in a standardised way. Perhaps assessment of adenosine-induced symptoms in combination with surrogate non-invasive indices would provide a better method for detecting hyperemia in patients receiving IV adenosine until alternative indices are developed.

\section{Study limitations}

This is a retrospectively analysed, heterogeneous cohort of patients who may have had different levels of pre-medication (including sedation), beta-blockade, tobacco smoking and caffeine intake prior to the catheter laboratory visit, confounding their responses to adenosine. However, at our institute, patients are advised to abstain from caffeine and anti-anginal medication, especially beta-blockers, prior to catheter laboratory tests.

Whilst an increase in coronary blood flow as determined by Doppler (CFR) is the gold standard for assessing hyperemia within the Catheter Laboratory, an absence of flow augmentation does not necessarily imply inadequate hyperemic stimulus. This is partly because CFR is dependent on the both epicardial vessels and microvasculature, hence may be an imperfect measure hyperemia, for example in the context of microvascular coronary disease where it may be impossible to distinguish inadequate hyperemic stimulus from diminished responsiveness. In patients who truly are non-responsive to adenosine, a different stressor such as Dobutamine or physical exercise may be more appropriate.

\section{Conclusion}

An increase in HR has high PPV but poor NPV as a surrogate marker of coronary hyperemia in response to IV adenosine. Even in patients who respond, the time to maximum HR is not always an indicator of maximal hyperemia. In the cardiac catheterisation laboratory, hyperemia is best adjudicated by assessing multiple coronary pressure waveform indices, including dicrotic notch disappearance, ventricularisation of the $\mathrm{Pd}$ trace and separation of $\mathrm{Pd}$ and $\mathrm{Pa}$ values, rather than reliance on one index alone. In the noninvasive setting, the high PPV of HR as a surrogate marker of hyperemia can be helpful, when no other reliable physiologic parameters are available, but should be interpreted cautiously. Further research is needed to develop optimal methods for identifying coronary hyperemia outside the catheter laboratory.

Acknowledgements The authors express appreciation to all members of the Cardiovascular Research Department and Catheter Laboratory at St Thomas' Hospital.

Funding Authors BM and HR are funded by British Heart Foundation Clinical Research Training Fellowship (FS/15/78/31678 \& FS/16/49/32320)

\section{Compliance with ethical standards}

Conflict of interest None of the authors have any conflict of interest that could have influenced this manuscript.

Ethical standards All human studies have been approved by the appropriate ethics committee and have therefore been performed in accordance with the ethical standards laid down in the 1964 Declaration of Helsinki and its later amendments.

Informed consent Informed consent was obtained from all individual participants included in the study.

Open Access This article is distributed under the terms of the Creative Commons Attribution 4.0 International License (http://creativeco mmons.org/licenses/by/4.0/), which permits unrestricted use, distribution, and reproduction in any medium, provided you give appropriate credit to the original author(s) and the source, provide a link to the Creative Commons license, and indicate if changes were made.

\section{References}

1. Hachamovitch R (2003) Comparison of the short-term survival benefit associated with revascularization compared with medical therapy in patients with no prior coronary artery disease undergoing stress myocardial perfusion single photon emission computed tomography. Circulation 107:2900-2907. https://doi. org/10.1161/01.CIR.0000072790.23090.41

2. Iskandrian AS, Heo J, Nguyen T et al (1991) Assessment of coronary artery disease using single-photon emission computed tomography with thallium-201 during adenosine-induced 
coronary hyperemia. Am J Cardiol 67:1190-1194. https://doi. org/10.1016/0002-9149(91)90925-B

3. De Bruyne B, Pijls NHJ, Kalesan B et al (2012) Fractional flow reserve-guided PCI versus medical therapy in stable coronary disease. New Engl J Med 367:991-1001. https://doi.org/10.1056/ NEJMoa1205361

4. Tonino PAL, De Bruyne B, Pijls NHJ et al (2009) Fractional flow reserve versus angiography for guiding percutaneous coronary intervention. New Engl J Med 360:213-224. https://doi. org/10.1056/NEJMoa0807611

5. Bech GJW, De Bruyne B, Pijls NHJ et al (2001) Fractional flow reserve to determine the appropriateness of angioplasty in moderate coronary stenosis: a randomized trial. Circulation 103:29282934. https://doi.org/10.1161/01.CIR.103.24.2928

6. Greenwood JP, Maredia N, Younger JF et al (2012) Cardiovascular magnetic resonance and single-photon emission computed tomography for diagnosis of coronary heart disease (CE-MARC): a prospective trial. Lancet 379:453-460. https://doi.org/10.1016/ S0140-6736(11)61335-4

7. Wilson RF, Wyche K, Christensen BV et al (1990) Effects of adenosine on human coronary arterial circulation. Circulation 82:1595-1606. https://doi.org/10.1161/01.CIR.82.5.1595

8. Dhalla AK, Wong M-Y, Wang W-Q et al (2006) Tachycardia caused by $\mathrm{A} 2 \mathrm{~A}$ adenosine receptor agonists is mediated by direct sympathoexcitation in awake rats. J Pharmacol Exp Ther 316:695-702. https://doi.org/10.1124/jpet.105.095323

9. Shryock JC, Belardinelli L (1997) Adenosine and adenosine receptors in the cardiovascular system: biochemistry, physiology, and pharmacology. Am J Cardiol 79:2-10. https://doi. org/10.1016/S0002-9149(97)00256-7

10. Karamitsos TD, Ntusi NA, Francis JM et al (2010) Feasibility and safety of high-dose adenosine perfusion cardiovascular magnetic resonance. J Cardiovasc Magn Reson 12:66. https://doi. org/10.1186/1532-429X-12-66

11. Kidambi A, Sourbron S, Maredia N et al (2015) Factors associated with false-negative cardiovascular magnetic resonance perfusion studies: a clinical evaluation of magnetic resonance imaging in coronary artery disease (CE-MARC) substudy. J Magn Reson Imaging 43:566-573. https://doi.org/10.1002/jmri.25032

12. Johnson NP, Johnson DT, Kirkeeide RL et al (2015) Repeatability of fractional flow reserve despite variations in systemic and coronary hemodynamics. JACC Cardiovasc Interv 8:1018-1027. https://doi.org/10.1016/j.jcin.2015.01.039

13. Siebes M (2010) Intracoronary doppler-based techniques for stenosis appraisal. Coronary stenosis imaging structure and physiology. PCR, Toulouse, pp. 377-400

14. Pacold I, Hwang MH, Piao ZE et al (1989) The mechanism and significance of ventricularization of intracoronary pressure during coronary angiography. Am Heart J 118:1160-1166

15. Wilkinson IB, MacCallum H, Flint L et al (2004) The influence of heart rate on augmentation index and central arterial pressure in humans. J Physiol 525:263-270. https://doi. org/10.1111/j.1469-7793.2000.t01-1-00263.x
16. Kramer CM, Barkhausen J, Flamm SD et al (2008) Standardized cardiovascular magnetic resonance imaging (CMR) protocols, society for cardiovascular magnetic resonance: board of trustees task force on standardized protocols. J Cardiovasc Magn Reson 10:35. https://doi.org/10.1186/1532-429X-10-35

17. Kern MJ, Deligonul U, Tatineni $S$ et al (1991) Intravenous adenosine: continuous infusion and low dose bolus administration for determination of coronary vasodilator reserve in patients with and without coronary artery disease. J Am Coll Cardiol 18:718-729

18. Alexopoulos D, Xanthopoulou I, Tsigkas G et al (2016) Effect of high $(200 \mu \mathrm{g} / \mathrm{kg}$ per minute) adenosine dose infusion on fractional flow reserve variability. J Am Heart Assoc 5:e004323. https://doi. org/10.1161/JAHA.116.004323

19. Agarwal SK, Raina S, Edupuganti M et al (2016) Predictive accuracy of resting gradient ( $\mathrm{pd} / \mathrm{pa}$ ) for identifying ischemic coronary lesions. J Am Coll Cardiol 67:380. https://doi.org/10.1016/S0735 -1097(16)30381-3

20. De Bruyne B, Pijls NHJ, Barbato E et al (2003) Intracoronary and intravenous adenosine 5'-triphosphate, adenosine, papaverine, and contrast medium to assess fractional flow reserve in humans. Circulation 107:1877-1883. https://doi.org/10.1161/01.CIR.00000 61950.24940 .88

21. Alberti C, Monopoli A, Casati C et al (1997) Mechanism and pressor relevance of the short-term cardiovascular and renin excitatory actions of the selective A2A-adenosine receptor agonists. J Cardiovasc Pharmacol 30:320-324. https://doi.org/10.1097/00005 344-199709000-00008

22. Biaggioni I, Killian TJ, Mosqueda-Garcia R et al (1991) Adenosine increases sympathetic nerve traffic in humans. Circulation 83:1668-1675. https://doi.org/10.1161/01.CIR.83.5.1668

23. Headrick JP, Peart JN, Reichelt ME et al (2011) Adenosine and its receptors in the heart: regulation, retaliation and adaptation. Biochim Biophys Acta (BBA) 1808:1413-1428. https://doi. org/10.1016/j.bbamem.2010.11.016

24. Nayeem MA, Zeldin DC, Boegehold MA et al (2010) Modulation by salt intake of the vascular response mediated through adenosine $\mathrm{A}(2 \mathrm{~A})$ receptor: role of CYP epoxygenase and soluble epoxide hydrolase. Am J Physiol Regul Integr Comp Physiol 299:R325-R333. https://doi.org/10.1152/ajpregu.00823.2009

25. Dixon AK, Gubitz AK, Sirinathsinghji DJS et al (2012) Tissue distribution of adenosine receptor mRNAs in the rat. Br J Pharmacol 118:1461-1468. https://doi.org/10.1111/j.1476-5381.1996. tb15561.x

26. Gessi S, Merighi S, Varani K et al (2008) The A3 adenosine receptor: an enigmatic player in cell biology. Pharmacol Ther 117:123-140. https://doi.org/10.1016/j.pharmthera.2007.09.002

27. Liu A, Wijesurendra RS, Ariga R et al (2017) Splenic T1-mapping: a novel quantitative method for assessing adenosine stress adequacy for cardiovascular magnetic resonance. J Cardiovasc Magn Reson 19:412

28. Johnson SG, Peters S (2010) Advances in pharmacologic stress agents: focus on regadenoson. J Nuclear Med Technol 38:163171. https://doi.org/10.2967/jnmt.109.065581 